\title{
Path integral for the Hilbert-Palatini and Ashtekar gravity
}

\author{
S.Yu. Alexandrov* \\ Department of Theoretical Physics, St.Petersburg University,198904 St.Petersburg, Russia
}

D.V. Vassilevich ${ }^{\dagger}$

Institute for Theoretical Physics, Leipzig University, Augustusplatz 10/11, 04109 Leipzig, Germany

\begin{abstract}
To write down a path integral for the Ashtekar gravity one must solve three fundamental problems. First, one must understand rules of complex contour functional integration with holomorphic action. Second, one should find which gauges are compatible with reality conditions. Third, one should evaluate the Faddeev-Popov determinant produced by these conditions. In the present paper we derive the BRST path integral for the Hilbert-Palatini gravity. We show, that for certain class of gauge conditions this path integral can be re-written in terms of the Ashtekar variables. Reality conditions define contours of integration. For our class of gauges all ghost terms coincide with what one could write naively just ignoring any Jacobian factors arising from the reality conditions.
\end{abstract}

PACS: 04.60.+n, 04.20.Fy

\section{INTRODUCTION}

Invention of complex canonical variables [1] opened a new avenue for non-perturbative treatment of quantum general relativity. In these new variables all constraints were made polynomial at the expense of introducing reality conditions. Afterwards, many gravitational theories were re-formulated in a similar way, including even eleven dimensional supergavity [2]. Quite spectacular success was achieved in loop quantum gravity [3]. In the view of recent progress of non-perturbative methods it seems especially important to develop the path integral formulation of the Ashtekar gravity which could serve as a bridge between perturbative and non-perturbative results.

Constraint structure of the Ashtekar gravity has been studied in some detail (for reviews, see [4] and [5]). The BRST charge was constructed [6]. However, this results are still insufficient for constructing a path integral. It is known, that any restriction imposed on integration variables may lead to the Faddeev-Popov ghosts [7]. It is unclear what kind of ghost action is induced by the reality conditions.

It is obvious that the path integral for the Ashtekar gravity will have a somewhat unusual form. In the case of complex scalar fields action is real and one integrates over whole complex plane. In the case of Ashtekar gravity action is holomorphic. Thus one may expect some sort of contour integration. Position of the contour must be defined by using the reality conditions. However, it is not known yet which gauges are compatible with these conditions.

Our strategy is rather simple. We derive the path integral for the Hilbert-Palatini gravity and than rewrite it in terms of the Ashtekar variables. By itself, the first part of our work is not a great novelty. Hamiltonian structure of

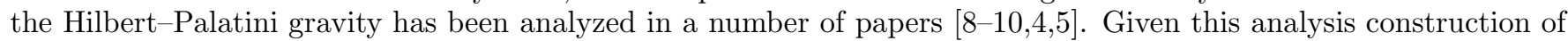
the path integral is quite straightforward. However, transition to the Ashtekar variables requires a complex canonical transformation which is not well defined in the path integral. We would also like to avoid any gauge fixing at intermediate steps before the path integral is written down. Thus we are forced to choose a basis in the HilbertPalatini action different from the ones used earlier and redo calculations of the constraint algebra, BRST charge, etc. A price to pay for the relatively easy transition to the Ashtekar variables in the path integral is an ugly form of the Hamiltonian constraint of the Hilbert-Palatini action. It leads to lengthy calculations at intermediate steps, which are reported here in some detail to make the paper self-contained.

As our main result, we transformed the Hilbert-Palatini path integral to the Ashtekar variables. This can be done successfully for a restricted class of gauges only. One is not allowed to impose gauge conditions on the connection variables. Therefore, path integral quantization of the Ashtekar gravity in an arbitrary gauge remains an open problem.

\footnotetext{
*e.mail: alexand@snoopy.phys.spbu.ru

${ }^{\dagger}$ Alexander von Humboldt fellow. On leave from: Department of Theoretical Physics, St.Petersburg University,198904 St.Petersburg, Russia. e.mail: Dmitri.Vassilevich@itp.uni-leipzig.de
} 
The paper is organized as follows. In the next section some preliminary information on the self dual Hilbert-Palatini action is collected. We introduce variables which will be convenient for construction of the path integral, re-derive the Ashtekar action and give some useful equations. In the third section we re-consider constraint structure of the Hilbert-Palatini gravity in terms of our variables. The fourth section is devoted to the BRST quantization of the Hilbert-Palatini gravity. In the section $\mathrm{V}$ we establish a relation between first and second class constraints of the Hilbert-Palatini action and the reality conditions and vanishing of imaginary part of the Ashtekar action. In the sixth section we re-write the path integral in terms of the Ashtekar variables. This represents our main result. The reader who do not want to go into technicalities of the BRST quantization will find a simple derivation of the Faddeev path integral for the Ashtekar gravity in section VII. In the last section some perspectives are briefly discussed. Technical details are collected in the Appendices.

\section{SELFDUAL HILBERT-PALATINI ACTION}

Let $\Omega^{\gamma \delta}=d \omega^{\gamma \delta}+\omega^{\gamma}{ }_{\alpha} \wedge \omega^{\alpha \delta}, \omega$ and $e$ are connection and tetrad one-forms respectively. Signature of the metric is $(-,+,+,+)$. The Levi-Civita tensor is defined by the equation $\varepsilon_{0123}=1$. Define the star operator as $\star \omega^{\alpha \beta}=$ $\frac{1}{2} \varepsilon^{\alpha \beta}{ }_{\gamma \delta} \omega^{\gamma \delta}$. Define

$$
\begin{aligned}
A^{\alpha \beta} & =\frac{1}{2}\left(\omega^{\alpha \beta}-i \star \omega^{\alpha \beta}\right) \\
\mathcal{F}^{\alpha \beta} & =d A^{\alpha \beta}+A_{\gamma}^{\alpha} \wedge A^{\gamma \delta}=\frac{1}{2}\left(\Omega^{\alpha \beta}-i \star \Omega^{\alpha \beta}\right)
\end{aligned}
$$

These fields satisfy $\star A=i A, \star \mathcal{F}=i \mathcal{F}$. Let us start with the selfdual Hilbert-Palatini action expressed in terms of selfdual connection only [10] 13 :

$$
S_{S D}=\int \varepsilon_{\alpha \beta \gamma \delta} e^{\alpha} \wedge e^{\beta} \wedge \mathcal{F}^{\gamma \delta}
$$

Let us split coordinates $x^{\mu}$ into "time" $t$ and "space" $x^{i}$ and introduce the notations:

$$
\begin{aligned}
e^{0} & =N d t+\chi_{a} E_{i}^{a} d x^{i}, \quad e^{a}=E_{i}^{a} d x^{i}+E_{i}^{a} N^{i} d t \\
A_{i}^{a} & =\varepsilon^{a b c} A_{b c i}, \quad A_{0}^{a}=\varepsilon^{a b c} A_{b c 0} \\
F_{i j}^{a} & =\varepsilon^{a b c} \mathcal{F}_{i j, b c}
\end{aligned}
$$

where $a, b, c=1,2,3$ are flat $S O(3)$ indices. $E_{a}^{i}$ will denote inverse of $E_{i}^{a}$. We also need weighted fields:

$$
\tilde{E}_{a}^{i}=\sqrt{h} E_{a}^{i}, \quad \underset{\sim}{N}=(\sqrt{h})^{-1} N
$$

$\sqrt{h}=\operatorname{det} E_{i}^{a}$. After long but elementary calculations we can represent (2) in the following form

$$
\begin{aligned}
S_{S D} & =2 \int d t d^{3} x\left(P_{a}^{i} \partial_{t} A_{i}^{a}+A_{0}^{a} \mathcal{G}_{a}+N^{i} \mathcal{H}_{i}+\underset{\sim}{N \mathcal{H}}\right), \\
P_{a}^{i} & =i\left(\widetilde{E}_{a}^{i}-i \varepsilon_{a}^{b c} \widetilde{E}_{b}^{i} \chi_{c}\right), \\
\mathcal{G}_{a} & =\nabla_{i} P_{a}^{i}=\partial_{i} P_{a}^{i}-\varepsilon_{a b c} A_{i}^{b} P^{c i}, \\
\mathcal{H}_{i} & =-2 i \widetilde{E}_{a}^{k} F_{i k}^{a}-\varepsilon_{i j k} \widetilde{E}_{a}^{j} \widetilde{E}_{b}^{k} \varepsilon^{l m n}{\underset{\sim}{E_{l}} \chi_{d} F_{m n}^{a b},}_{\mathcal{H}}=2 \widetilde{E}_{a}^{i} \widetilde{E}_{b}^{k} F_{i k}^{a b}
\end{aligned}
$$

$\underset{\sim}{E_{i}^{a}}=h^{-1 / 2} E_{i}^{a}$. By a suitable redefinition of Lagrange multipliers $\chi^{a}$ can be removed from the action.

$$
\mathcal{N}_{D}^{i}=N^{i}+\frac{\tilde{E}_{a}^{i} \chi^{a}\left(N^{j} \underset{\sim}{\left.E_{j}^{b} \chi_{b}-\underset{\sim}{\sim}\right)}\right.}{1-\chi^{2}} \quad \underset{\sim}{\mathcal{N}}=\frac{\underset{\sim}{N}-N^{i}{\underset{\sim}{E}}_{i}^{a} \chi_{a}}{1-\chi^{2}}
$$

The action (5) now reads: 


$$
\begin{aligned}
S_{S D}=S_{A} & =2 \int d t d^{3} x\left(P_{a}^{i} \partial_{t} A_{i}^{a}+A_{0}^{a} \mathcal{G}_{a}+\mathcal{N}_{D}^{i} H_{i}+\underset{\sim}{\mathcal{N} H}\right) \\
H_{i} & =-2 P_{a}^{k} F_{i k}^{a} \\
H & =-2 P_{a}^{i} P_{b}^{k} F_{i k}^{a b}
\end{aligned}
$$

All $\chi$-dependence is hidden in the canonical variables. We arrived at the Ashtekar action (7) (later denoted as $S_{A}$ ). Absence of $\chi$ in $S_{A}$ leads to a first class primary constraint $p_{\chi}=0$, where $p_{\chi}$ is canonical momentum for $\chi$. This constraint generates shifts of $\chi$ by an arbitrary function and originates from the Lorentz boosts.

One must bear in mind that not all the components of $\operatorname{Re} P_{a}^{i}$ are independent. To restore correct form of $P_{a}^{i}$ one needs a condition $\operatorname{Im} P_{a}^{(i} \operatorname{Re} P_{a}^{j)}=0$ or, equivalently,

$$
\operatorname{Im}\left(P_{a}^{i} P_{a}^{j}\right)=0
$$

The equation (8) is known as first metric reality condition. Being supplemented by the second metric reality condition

$$
\partial_{t} \operatorname{Im}\left(P_{a}^{i} P_{a}^{j}\right)=0
$$

on an initial hypersurface it ensures real evolution of the metric [14 16]. As usual, the triad field $\widetilde{E}$ should be non-degenerate.

Define the smeared constraints:

$$
\begin{aligned}
& \mathcal{G}(n)=\int d^{3} x n^{a} \mathcal{G}_{a}, \quad H^{A}(\underset{\sim}{N})=\int d^{3} x \underset{\sim}{N} H \\
& \mathcal{D}(\vec{N})=\int d^{3} x N^{i}\left(H_{i}+2 A_{i}^{a} \mathcal{G}_{a}\right),
\end{aligned}
$$

They obey the following algebra:

$$
\begin{aligned}
& \{\mathcal{G}(n), \mathcal{G}(m)\}_{C}=-\mathcal{G}(n \times m), \\
& \{\mathcal{D}(\vec{N}), \mathcal{D}(\vec{M})\}_{C}=-2 \mathcal{D}([\vec{N}, \vec{M}]), \\
& \{\mathcal{D}(\vec{N}), \mathcal{G}(n)\}_{C}=-2 \mathcal{G}\left(N^{i} \partial_{i} n\right), \\
& \left\{H^{A}(\underset{\sim}{N}), \mathcal{G}(n)\right\}_{C}=0 \\
& \left\{\mathcal{D}(\vec{N}), H^{A}(\underset{\sim}{N})\right\}_{C}=-2 H^{A}\left(\mathcal{L}_{\vec{N}} \stackrel{N}{\sim}\right), \\
& \left\{H^{A}(\underset{\sim}{N}), H^{A}(\underset{\sim}{M})\right\}_{C}=2 \mathcal{D}(\vec{K})-2 \mathcal{G}\left(2 K^{j} A_{j}\right)
\end{aligned}
$$

where

$$
\begin{aligned}
& (n \times m)^{a}=\varepsilon^{a b c} n^{b} m^{c}, \quad \mathcal{L}_{\vec{N}} N=N^{i} \partial_{i} \underset{\sim}{N}-\underset{\sim}{N} \partial_{i} N^{i}, \\
& {[\vec{N}, \vec{M}]^{i}=N^{k} \partial_{k} M^{i}-M^{k} \partial_{k} N_{i},} \\
& K^{j}=\left(\underset{\sim}{N} \partial_{i} \sim_{\sim}^{M}-\underset{\sim}{M} \partial_{i} \underset{\sim}{N}\right) P_{a}^{i} P_{a}^{j}
\end{aligned}
$$

We introduced the subscript $C$ to distinguish the Poisson bracket $\{\cdot, \cdot\}_{C}$ of the complex Ashtekar theory from that of the real Hilbert-Palatini action.

\section{HAMILTONIAN FORM OF THE HILBERT-PALATINI ACTION}

Let us start with the Hilbert-Palatini action 


$$
S=\frac{1}{2} \int \varepsilon_{\alpha \beta \gamma \delta} e^{\alpha} \wedge e^{\beta} \wedge \Omega^{\gamma \delta}
$$

Recall that the Ashtekar action is obtained from the Hilbert-Palatini one by adding a pure imaginary term $-i \frac{1}{2} \int \varepsilon_{\alpha \beta \gamma \delta} e^{\alpha} \wedge e^{\beta} \wedge \star \Omega^{\gamma \delta}$. Therefore,

$$
S=\operatorname{Re} S_{A}=2 \int d t d^{3} x\left(\widetilde{E}_{a}^{i} \partial_{t} \omega_{i}^{0 a}+Z_{a}^{i} \partial_{t} \xi_{i}^{a}+n_{G}^{a} \operatorname{Re} \mathcal{G}_{a}+n_{L}^{a} \operatorname{Im} \mathcal{G}_{a}+\mathcal{N}_{D}{ }^{i} \operatorname{Re} H_{i}+\underset{\sim}{\mathcal{N}} \operatorname{Re} H\right)
$$

where

$$
\begin{aligned}
n_{G}^{a} & =\operatorname{Re} A_{0}^{a}, \quad n_{L}^{a}=-\operatorname{Im} A_{0}^{a} \\
Z_{a}^{i} & =\varepsilon_{a}^{b c} \widetilde{E}_{b}^{i} \chi_{c} \\
\xi_{i}^{a} & =\frac{1}{2} \varepsilon^{a}{ }_{b c} \omega_{i}^{b c}
\end{aligned}
$$

In order to simplify the constraint algebra we replace $R e H_{i}$ by the modified vector constraint. To this end we shift the Lagrange multipliers.

$$
n_{G}^{a}=\mathcal{N}_{G}^{a}+2 \mathcal{N}_{D}^{i} \xi_{i}^{a} \quad n_{L}^{a}=\mathcal{N}_{L}^{a}+2 \mathcal{N}_{D}^{i} \omega_{i}^{0 a}
$$

We see that $\tilde{E}_{a}^{i}$ plays a role of the momentum for $\xi_{i}^{a}$ whereas $Z_{a}^{i}$ is momentum conjugate to $\omega_{i}^{0 a}$. $Z_{a}^{i}$ has three independent components only. To have time derivatives of true dynamical variables we replace

$$
\omega_{i}^{0 a}=\eta_{i}^{a}+\varepsilon^{a b c} \xi_{i}^{b} \chi_{c}
$$

Then the kinetic term reads $\widetilde{E}_{a}^{i} \partial_{t} \eta_{i}^{a}-\left(\varepsilon^{a b c} \xi_{i}^{b} \widetilde{E}_{c}^{i}\right) \partial_{t} \chi_{a}$. By a suitable change of variables we can bring this term to the standard form $p \partial_{t} q$. Let us introduce a basis in the space of $3 \times 3$ matrices.

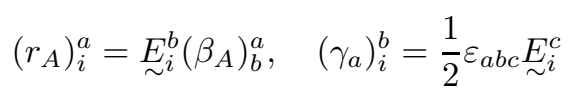

where $\beta_{A}$ are six symmetric $3 \times 3$ matrices. Define

$$
\xi_{i}^{a}=r_{i}^{a}+\left(\gamma_{b}\right)_{i}^{a} \omega^{b}, \quad r_{i}^{a}=\left(r_{A}\right)_{i}^{a} \lambda^{A}
$$

$\omega$ and $\lambda$ will be treated as new canonical variables.

We arrive at the following expression for the Hilbert-Palatini action

$$
\begin{aligned}
& \frac{1}{2} S=\int d t d^{3} x\left(\widetilde{E}_{a}^{i} \partial_{t} \eta_{i}^{a}+\chi_{a} \partial_{t} \omega^{a}+\mathcal{N}_{G}^{a} \Phi_{a}^{G}+\mathcal{N}_{L}^{a} \Phi_{a}^{L}+\mathcal{N}_{D}^{i} \Phi_{i}^{D}+\underset{\sim}{\mathcal{N}} \Phi^{H}\right) \\
& \Phi_{a}^{G}=\partial_{i}\left(\varepsilon_{a}{ }^{b c} \tilde{E}_{b}^{i} \chi_{c}\right)-\varepsilon_{a b}{ }^{c} \eta_{i}^{b} \tilde{E}_{c}^{i}-\varepsilon_{a b}{ }^{c} \omega^{b} \chi_{c} \\
& \Phi_{a}^{L}=\partial_{i} \widetilde{E}_{a}^{i}+\varepsilon_{a b c} \eta_{i}^{b} \varepsilon^{c g f} \widetilde{E}_{g}^{i} \chi_{f}-\left(\delta_{a b}-\chi_{a} \chi_{b}\right) \omega^{b} \\
& \Phi_{i}^{D}=-2\left[\tilde{E}_{a}^{j} \partial_{i} \eta_{j}^{a}-\partial_{j}\left(\tilde{E}_{a}^{j} \eta_{i}^{a}\right)-\omega^{a} \partial_{i} \chi_{a}\right] \\
& \Phi^{H}=\varepsilon^{a b c} \tilde{E}_{b}^{i} \tilde{E}_{c}^{j}\left(\delta_{a d}-\chi_{a} \chi_{d}\right) \varepsilon_{g f}^{d} \eta_{i}^{g} \eta_{j}^{f}+2 \tilde{E}_{a}^{i} \widetilde{E}_{b}^{j} \chi^{b}\left(\partial_{i} \eta_{j}^{a}-\partial_{j} \eta_{i}^{a}\right) \\
& -\left(1-\chi^{2}\right)\left(2 \partial_{i}\left(\tilde{E}_{a}^{i} \omega^{a}\right)-h^{-1} \omega^{a} \partial_{i}\left(h \tilde{E}_{a}^{i}\right)\right)+\omega^{a} \chi^{b}\left(\widetilde{E}_{a}^{i} \partial_{i} \chi^{b}+\tilde{E}_{b}^{i} \partial_{i} \chi_{a}\right) \\
& +\tilde{E}_{a}^{j} \omega^{b}\left(\chi_{a} \eta_{j}^{b}-\chi_{b} \eta_{j}^{a}\right)-\omega^{a} \chi_{a}\left(\tilde{E}_{b}^{j} \eta_{j}^{c} \chi^{b} \chi_{c}-\chi^{2} \widetilde{E}_{b}^{j} \eta_{j}^{b}\right) \\
& -\frac{1}{2}\left(1-\chi^{2}\right) \omega^{a} \omega^{b}\left(\delta_{a b}-\chi_{a} \chi_{b}\right) \\
& +2 \varepsilon^{a b c} \widetilde{E}_{b}^{i} \tilde{E}_{c}^{j}\left(\left(1-\chi^{2}\right) \partial_{i} r_{j}^{a}+r_{j}^{d} \chi_{d} \partial_{i} \chi_{a}-\left(1-\chi^{2}\right) \chi_{a} r_{j}^{d} \eta_{i}^{d}\right. \\
& \left.+\left(\delta_{a g}-\chi_{a} \chi_{g}\right) \eta_{i}^{g} r_{j}^{d} \chi_{d}\right)-\left(1-\chi^{2}\right) \varepsilon^{a b c} \tilde{E}_{b}^{i} \tilde{E}_{c}^{j}\left(\delta_{a d}-\chi_{a} \chi_{d}\right) \varepsilon^{d}{ }_{g f} r_{i}^{g} r_{j}^{f}
\end{aligned}
$$

We see that $\lambda_{A}$ has no conjugate momentum, and thus is non-dynamical. We observe also that $\lambda_{A}$ is contained in $\Phi^{H}$ only.

Let us analyse constraints of the theory along the lines of usual Dirac procedure [17. Since all steps are completely standard we omit irrelevant technical details (cf. 10,何). First we note that $\widetilde{E}_{a}^{i}$ and $\chi_{a}$ are conjugate momenta to $\eta_{i}^{a}$ and $\omega^{a}$ respectively. By analyzing the consistency conditions we get the following set of constraints 


$$
p_{\alpha}^{(n)}=0 \quad p_{A}^{(\lambda)}=0 \quad \Phi_{\alpha}=0 \quad \mathcal{N} \frac{\partial \Phi^{H}}{\partial \lambda_{A}}=0
$$

where $p^{(q)}$ denotes momentum conjugate to variable $q,(n)$ are all Lagrange multipliers, and $\Phi_{\alpha}=\left(\Phi_{a}^{G}, \Phi_{a}^{L}, \Phi_{i}^{D}, \Phi^{H}\right)$. Introduce

$$
\Phi_{\alpha}^{\prime}=\Phi_{\alpha}-\frac{1}{2} p_{A}^{\lambda} \mathcal{A}_{A B}^{-1}\left\{\Phi_{\alpha}, \frac{\partial \Phi^{H}}{\partial \lambda_{B}}\right\}
$$

where $\mathcal{A}_{A B}=-\frac{1}{2} \frac{\partial^{2} \Phi^{H}}{\partial \lambda_{A} \partial \lambda_{B}}$. Then $\Phi_{\alpha}^{\prime}$ and $p_{\alpha}^{(n)}$ are first class constraints.

Remaining constraints $p_{A}^{(\lambda)}$ and $\underset{\sim}{\mathcal{N}} \frac{\partial \Phi^{H}}{\partial \lambda_{A}}$ are second class constraints with nontrivial matrix of commutators. This matrix is non-degenerate and can be used to construct Dirac's bracket. To avoid using such an object one should solve second class constraints explicitly.

The constraints $p_{A}^{(\lambda)}=0$ are solved trivially giving us back $\Phi_{\alpha}$ as first class constraints. Since $\Phi^{H}$ is quadratic in $\lambda$, it can be represented as

$$
\Phi^{H}=\Phi_{0}^{H}+2 \mathcal{B}_{A} \lambda_{A}-\lambda_{A} \mathcal{A}_{A B} \lambda_{B},
$$

The remaining second class constraints give the equations

$$
0=\frac{\delta \Phi^{H}}{\delta \lambda^{A}}=2\left(-\mathcal{A}_{A B} \lambda_{B}+\mathcal{B}_{A}\right)
$$

which can be solved for $\lambda$ resulting in expressions for non-dynamical components $r_{i}^{a}$ in terms of other canonical variables. Here we give final results only. Some intermediate steps are reported in the Appendix A.

$$
\begin{aligned}
& r_{i}^{a}=\frac{1}{2\left(1-\chi^{2}\right)}\left(-X_{a d} \varepsilon^{d b c} \widetilde{E}_{b}^{k} \widetilde{E}_{c}^{j} X_{g f} \underset{\sim}{E_{i}^{g}} \partial_{k} \underset{\sim j}{E_{j}^{f}}\right.
\end{aligned}
$$

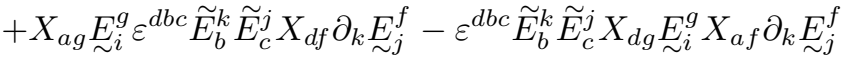

$$
\begin{aligned}
& -\chi_{a} \varepsilon_{d b c} \widetilde{E}_{b}^{j} X_{c g} E_{\sim}^{g} \partial_{i} \chi_{d}+\varepsilon^{a b c} \chi_{b} \partial_{i} \chi_{c}-\varepsilon^{a b c} \chi_{b} \widetilde{E}_{c}^{j} E_{\sim}^{d} \partial_{j} \chi_{d} \\
& \left.+\varepsilon^{a b c} \chi_{b} \eta_{i}^{c}+\varepsilon^{d b c} \tilde{E}_{a}^{j}{\underset{\sim}{d i}}_{i}^{d} \chi_{b} \eta_{j}^{c}\right),
\end{aligned}
$$

where $X_{a b}=\left(\delta_{a b}-\chi_{a} \chi_{b}\right)$. The Hamiltonian constraint reads:

$$
\begin{aligned}
& \Phi^{H}=\Phi_{0}^{H}+\mathcal{B}_{A} \mathcal{A}_{A B}^{-1} \mathcal{B}_{B} \\
& =-\frac{1}{2}\left(1-\chi^{2}\right) \omega^{a} \omega^{b} X_{a b}-\left(1-\chi^{2}\right)\left(2 \partial_{i}\left(\widetilde{E}_{a}^{i} \omega^{a}\right)-\right. \\
& \left.h^{-1} \omega^{a} \partial_{i}\left(h \widetilde{E}_{a}^{i}\right)\right)+\omega^{a} \chi_{b}\left(\widetilde{E}_{a}^{i} \partial_{i} \chi_{b}+\widetilde{E}_{b}^{i} \partial_{i} \chi_{a}\right) \\
& +\left(\widetilde{E}_{a}^{i} \omega^{b}\left(\chi_{a} \eta_{i}^{b}-\chi_{b} \eta_{i}^{a}\right)-\omega^{a} \chi_{a}\left(\widetilde{E}_{b}^{i} \chi^{b} \eta_{i}^{c} \chi_{c}-\chi^{2} \widetilde{E}_{b}^{i} \eta_{i}^{b}\right)\right) \\
& +\frac{1}{2}\left\{-\varepsilon^{a b c} \widetilde{E}_{b}^{i} \widetilde{E}_{c}^{j} X_{a d} \varepsilon^{d p q} \widetilde{E}_{p}^{k} \widetilde{E}_{q}^{l} X_{g f} \partial_{i}{\underset{\sim}{E_{j}}}_{g} \partial_{k} \underset{\sim l}{E_{l}^{f}+}\right. \\
& \varepsilon^{a b c} \widetilde{E}_{b}^{i} \widetilde{E}_{c}^{j} X_{a g} \partial_{i} \underset{\sim j}{E_{j}^{g}} \varepsilon^{d p q} \widetilde{E}_{p}^{k} \widetilde{E}_{q}^{l} X_{d f} \partial_{k} \underset{\sim l}{E_{l}^{f}} \\
& \left.-\varepsilon^{a b c} \widetilde{E}_{b}^{i} \widetilde{E}_{c}^{j} X_{a g} \partial_{k} \underset{\sim l}{E_{l}^{g}} \varepsilon^{d p q} \widetilde{E}_{p}^{k} \widetilde{E}_{q}^{l} X_{d f} \partial_{i}{\underset{\sim}{j}}_{j}^{f}\right\} \\
& +\left\{-\varepsilon^{a b c} \widetilde{E}_{b}^{i} \widetilde{E}_{c}^{j} \chi_{a} \varepsilon^{d p q} \widetilde{E}_{p}^{k} \partial_{k} \chi_{d} X_{q g} \partial_{i}{\underset{\sim}{j}}_{j}^{g}+\varepsilon^{a b c} \widetilde{E}_{b}^{i} \widetilde{E}_{c}^{j} \partial_{i}{\underset{\sim}{E}}_{j}^{a} \varepsilon^{d p q} \widetilde{E}_{p}^{k} \partial_{k}{\underset{\sim}{f}}_{l}^{f} \chi_{q}\right. \\
& \left.-\varepsilon^{a b c} \tilde{E}_{b}^{i} \tilde{E}_{c}^{j} \varepsilon^{a d p} \tilde{E}_{p}^{k} \partial_{k} \chi_{d} \partial_{i}{\underset{\sim}{E_{j}} \chi_{g}}_{\}}\right\} \\
& -\frac{\chi^{2}}{2\left(1-\chi^{2}\right)} \varepsilon^{a b c} \tilde{E}_{b}^{i} \partial_{i} \chi_{a} X_{c q} \varepsilon^{d p q} \tilde{E}_{p}^{j} \partial_{j} \chi_{d} \\
& +\varepsilon^{a b c} \widetilde{E}_{a}^{k} \widetilde{E}_{b}^{i} \widetilde{E}_{c}^{j} \varepsilon^{d p q} \chi_{d} \eta_{k}^{p} \partial_{i}{\underset{\sim}{E_{j}}}^{q}-\varepsilon^{a b c} \widetilde{E}_{b}^{i} \partial_{i} \widetilde{E}_{c}^{j} \varepsilon^{a p q} \chi_{p} \eta_{j}^{q} \\
& +\frac{1}{1-\chi^{2}} \varepsilon^{a b c} \widetilde{E}_{b}^{i} \partial_{i} \chi_{a} \varepsilon^{c p q} \chi_{p} \eta_{j}^{q} \widetilde{E}_{d}^{j} \chi_{d} \\
& +\left\{2 \widetilde{E}_{a}^{i} \widetilde{E}_{b}^{j} \chi_{b}\left(\partial_{i} \eta_{j}^{a}-\partial_{j} \eta_{i}^{a}\right)+\varepsilon^{a b c} \widetilde{E}_{b}^{i} \widetilde{E}_{c}^{j} \varepsilon_{a p q} \eta_{i}^{p} \eta_{j}^{q}+\frac{1}{2} \varepsilon^{a b c} \chi_{a} \widetilde{E}_{b}^{i} \eta_{j}^{c} \varepsilon^{d p q} \chi_{d} \widetilde{E}_{p}^{j} \eta_{i}^{q}\right. \\
& \left.-\frac{1}{2} \varepsilon^{a b c} \chi_{a} \eta_{i}^{b} \varepsilon^{c p q} \chi_{p} \eta_{j}^{q} \widetilde{E}_{g}^{i} \widetilde{E}_{g}^{j}-\frac{1}{2\left(1-\chi^{2}\right)} \varepsilon^{a b c} \chi_{a} \eta_{i}^{b} \varepsilon^{c p q} \chi_{p} \eta_{j}^{q} \widetilde{E}_{g}^{i} \chi_{g} \widetilde{E}_{f}^{j} \chi_{f}\right\} .
\end{aligned}
$$


We end up this section with some useful commutators. Introduce smeared first class constraints:

$$
\begin{aligned}
& G(n)=\int d^{3} x n^{a} \Phi_{a}^{G}, \quad L(m)=\int d^{3} x m^{b} \Phi_{b}^{L}, \\
& \left.D(\vec{N})=\int d^{3} x N^{i} \Phi_{i}^{D}, \quad H \underset{\sim}{N}\right)=\int d^{3} x \underset{\sim}{N} \Phi^{H}
\end{aligned}
$$

Here all the constraints are taken from (23), except for the Hamiltonian constraint $\Phi^{H}$ which is now given by $(29)$. $\xi_{i}^{a}$ is expressed in terms of canonical variables by means of (22) and (28).

The transformations of the connection fields are:

$$
\begin{aligned}
& \left\{G(n), \xi_{j}^{d}\right\}=\varepsilon^{d a b} n^{a} \xi_{j}^{b}+\partial_{j} n^{d}, \\
& \left\{G(n), \eta_{j}^{d}+\varepsilon^{d p q} \xi_{j}^{p} \chi_{q}\right\}=\varepsilon^{d a b} n^{a}\left(\eta_{j}^{b}+\varepsilon^{b p q} \xi_{j}^{p} \chi_{q}\right), \\
& \left\{L(m), \xi_{j}^{d}\right\}=-\varepsilon^{d a b} m^{a}\left(\eta_{j}^{b}+\varepsilon^{b p q} \xi_{j}^{p} \chi_{q}\right), \\
& \left\{L(m), \eta_{j}^{d}+\varepsilon^{d p q} \xi_{j}^{p} \chi_{q}\right\}=\varepsilon^{d a b} m^{a} \xi_{j}^{b}+\partial_{j} m^{d}, \\
& \left\{D(\vec{N}), \xi_{j}^{d}\right\}=2\left(N^{i} \partial_{i} \xi_{j}^{d}+\xi_{i}^{d} \partial_{j} N^{i}\right), \\
& \left\{D(\vec{N}), \eta_{j}^{d}+\varepsilon^{d p q} \xi_{j}^{p} \chi_{q}\right\}=2\left(N^{i} \partial_{i}\left(\eta_{j}^{d}+\varepsilon^{d p q} \xi_{j}^{p} \chi_{q}\right)+\left(\eta_{i}^{d}+\varepsilon^{d p q} \xi_{i}^{p} \chi_{q}\right) \partial_{j} N^{i}\right)
\end{aligned}
$$

The Poisson brackets between the constraints are straightforward to evaluate. One obtains

$$
\begin{aligned}
& \{G(n), G(m)\}=-G(n \times m), \\
& \{L(n), L(m)\}=G(n \times m), \\
& \{G(n), L(m)\}=-L(n \times m), \\
& \{D(\vec{N}), D(\vec{M})\}=-2 D([\vec{N}, \vec{M}]), \\
& \{D(\vec{N}), G(n)\}=-2 G\left(N^{i} \partial_{i} n\right), \\
& \{D(\vec{N}), L(m)\}=-2 L\left(N^{i} \partial_{i} m\right), \\
& \{H(\underset{\sim}{N}), G(n)\}=0, \\
& \{H(\underset{\sim}{N}), L(m)\}=0, \\
& \{D(\vec{N}), H(\underset{\sim}{N})\}=-2 H\left(\mathcal{L}_{\vec{N}} N\right), \\
& \{H(\underset{\sim}{N}), H(\underset{\sim}{\sim})\}=2 D(\vec{K})-2 G\left(2 K^{j} \xi_{j}\right)-2 L\left(2 K^{j}\left(\eta_{j}+\xi_{j} \times \chi\right)\right)
\end{aligned}
$$

where

$$
\begin{aligned}
& K^{j}[\underset{\sim}{N}, \underset{\sim}{M}]=\left(\underset{\sim}{N} \partial_{i} \underset{\sim}{M}-\underset{\sim}{M} \partial_{i} \underset{\sim}{N}\right) K^{i j} \\
& K^{i j}=-\left(\widetilde{E}_{a}^{i} \widetilde{E}_{a}^{j}\left(1-\chi^{2}\right)+\widetilde{E}_{a}^{i} \chi_{a} \widetilde{E}_{b}^{j} \chi_{b}\right) .
\end{aligned}
$$

Other notations are taken from (12). $K^{i}$ is in fact the same as in (13) but written in different variables.

$\Phi^{H}$ will be called the Hamiltonian constraint. $\Phi^{D}$ generates diffeomorphisms of the 3 -surface and will be called the diffeomorphism constraint. $\Phi^{G}$ and $\Phi^{L}$ generate the $S O(3, R)$ rotations and the Lorentz boosts respectively. They will be called the Gauss law constraint and the Lorentz constraint, respectively. 
There is a set of remarkable relations between the Poisson brackets of the Hilbert-Palatini gravity and that of the Ashtekar gravity.

$$
\begin{aligned}
& \left\{\mathcal{G}(n), P_{a}^{j}\right\}_{C}=\left\{G(n), P_{a}^{j}\right\}=\left\{i L(n), P_{a}^{j}\right\}, \\
& \left\{\mathcal{G}(n), A_{j}^{a}\right\}_{C}=\left\{G(n), A_{j}^{a}\right\}=\left\{i L(n), A_{j}^{a}\right\}, \\
& \left\{\mathcal{D}(\vec{N}), P_{a}^{j}\right\}_{C}=\left\{D(\vec{N}), P_{a}^{j}\right\}, \quad\left\{\mathcal{D}(\vec{N}), A_{j}^{a}\right\}_{C}=\left\{D(\vec{N}), A_{j}^{a}\right\}, \\
& \left\{H^{A}(N), P_{a}^{j}\right\}_{C}=\left\{H(N), P_{a}^{j}\right\}
\end{aligned}
$$

Note, that last relation holds for $P_{a}^{j}$ only.

In a different context relation between Hilbert-Palatini and Ashtekar brackets was considered recently by Khatsymovsky [18].

\section{BRST QUANTIZATION OF THE HILBERT-PALATINI GRAVITY}

In this section we construct the BRST path integral [19] for the Hilbert-Palatini gravity. Here we follow the review [20]. Consider a dynamical system with phase space variables $\left(q^{s}, p_{s}\right)$, Hamiltonian $H_{0}$, and constraints $\Phi_{\alpha}$. Let $n^{\alpha}$ be the Lagrange multipliers associated with the constraints $\Phi_{\alpha}$, and $\pi_{\alpha}$ be the canonically conjugate momenta. The extended phase space is defined by introducing extra ghost and antighost fields $\left(b^{\alpha}, \bar{c}_{\alpha}, c^{\alpha}, \bar{b}_{\alpha}\right)$. obeying the following nonvanishing antibrackets

$$
\left\{b^{\alpha}, \bar{c}_{\beta}\right\}_{+}=-\delta_{\beta}^{\alpha},\left\{c^{\alpha}, \bar{b}_{\beta}\right\}_{+}=-\delta_{\beta}^{\alpha}
$$

$c^{\alpha}, \bar{c}_{\alpha}$ are real, whereas $b^{\alpha}, \bar{b}_{\alpha}$ are imaginary.

It is convenient to define an additional structure on the extended phase space, that of "ghost number". This is done by attributing the following ghost number to the canonical variables: $c^{\alpha}, b^{\alpha}$ have ghost number one, $\bar{c}_{\alpha}, \bar{b}_{\alpha}$ have ghost number minus one. All other variables have ghost number zero.

On this space one can construct a BRST generator $\Omega$ and a BRST invariant Hamiltonian $H$. They are determined by the following conditions:

(a) $\Omega$ is real and odd; (b) $\Omega$ has ghost number one; (c) $\Omega=-i b^{\alpha} \pi_{\alpha}+c^{\alpha} \Phi_{\alpha}+"$ higher ghost terms"; (d) $\{\Omega, \Omega\}+=0$

(a) $H$ is real and even; (b) $H$ has ghost number zero; (c) $H$ coincides with $H_{0}$ up to higher ghost terms; (d) $\{H, \Omega\}=0$

If $H_{0}$ weakly vanishes (as in our case) one can take $H=0$ since the formalism supports an arbitrariness in the definition of observables: $H_{0} \sim H_{0}+k^{\alpha} \Phi_{\alpha}$.

The BRST generator is fully defined by structure functions of the constraint algebra:

$$
\Omega=-i b^{\alpha} \pi_{\alpha}+\sum_{n \geq 0} c^{\alpha_{n+1}} \cdots c^{\alpha_{1}} U_{\alpha_{1} \cdots \alpha_{n+1}}^{(n) \beta_{1} \cdots \beta_{n}} \bar{b}_{\beta_{n}} \cdots \bar{b}_{\beta_{1}}
$$

The structure functions for the Hilbert-Palatini gravity are constructed in the Appendix B. As a result, we obtain

$$
\Omega=-i b^{\alpha} \pi_{\alpha}+c^{\alpha} \Phi_{\alpha}+\frac{1}{2} c^{\alpha} c^{\beta} C_{\alpha \beta}^{\gamma} \bar{b}_{\gamma}+c^{\alpha} c^{\beta} c^{\gamma} U_{\alpha \beta \gamma}^{(2) \delta \lambda} \bar{b}_{\delta} \bar{b}_{\lambda}
$$

where $U^{(2)}$ is taken from $(\overline{\mathrm{B} 8})$. Note that for the Yang-Mills theory the term with $U^{(2)}$ is absent in the BRST charge. This is also the case of the Ashtekar gravity [6].

The quantization is based on the generating functional for the Green functions which is represented in the form

$$
Z[j, J, \lambda]=\int \mathcal{D} \mu e^{i \int d t\left(L_{e f f}+j_{s} q^{s}+J^{s} p_{s}+\lambda_{\alpha} n^{\alpha}\right)}
$$

where

$$
L_{e f f}=\dot{q}^{s} p_{s}+\dot{n}^{\alpha} \pi_{\alpha}+\dot{c}^{\alpha} \bar{b}_{\alpha}+\dot{b}^{\alpha} \bar{c}_{\alpha}-H_{e f f} \quad H_{e f f}=H-\{\psi, \Omega\}_{+}
$$

Here $\psi$ is an odd and imaginary function which has ghost number minus one and plays a role of gauge fixing function, whereas $\mathcal{D} \mu$ is the usual measure (product over time of the Liouville measure of the extended phase space).

Let us choose 


$$
\psi=-\bar{b}_{\alpha} n^{\alpha}+i \bar{c}_{\alpha}\left(\frac{1}{\gamma} f^{\alpha}(q, p)+\frac{1}{\gamma} g^{\alpha}(n)\right) .
$$

By substituting (35) and (38) in (37) and putting $H=0$ one obtains:

$$
\begin{aligned}
H_{e f f}= & -n^{\alpha} \Phi_{\alpha}-i \bar{b}_{\alpha} b^{\alpha}+c^{\alpha} n^{\beta} C_{\alpha \beta}^{\gamma} \bar{b}_{\gamma}-3 c^{\alpha} c^{\beta} n^{\gamma} U_{\alpha \beta \gamma}^{(2) \delta \lambda} \bar{b}_{\delta} \bar{b}_{\lambda} \\
& +\frac{1}{\gamma}\left\{\left(f^{\alpha}+g^{\alpha}\right) \pi_{\alpha}-\bar{c}_{\alpha} \frac{\partial g^{\alpha}}{\partial n^{\beta}} b^{\beta}-i \bar{c}_{\alpha}\left\{f^{\alpha}, \Phi_{\beta}\right\} c^{\beta}-i \bar{c}_{\alpha}\left\{f^{\alpha}, C_{\beta \gamma}^{\delta}\right\} c^{\beta} c^{\gamma} \bar{b}_{\delta}\right. \\
& \left.-i \bar{c}_{\alpha}\left\{f^{\alpha}, U_{\beta \gamma \delta}^{(2) \xi \eta}\right\} c^{\beta} c^{\gamma} c^{\delta} \bar{b}_{\xi} \bar{b}_{\eta}\right\}
\end{aligned}
$$

Let us make the change of variables with unit Jacobian:

$$
\pi_{\alpha} \longrightarrow \gamma \pi_{\alpha}, \bar{c}_{\alpha} \longrightarrow \gamma \bar{c}_{\alpha}
$$

Then let $\gamma \longrightarrow 0$. In this limit integration over $\pi_{\alpha}, b^{\alpha}$ and $\bar{b}_{\alpha}$ is easily performed giving:

$$
Z[j, J, \lambda]=\int \mathcal{D} q \mathcal{D} p \mathcal{D} n \mathcal{D} c \mathcal{D} \bar{c} \delta\left(f^{\alpha}+g^{\alpha}\right) e^{i \int d t\left(L_{e f f}^{\prime}+j_{s} q^{s}+J^{s} p_{s}+\lambda_{\alpha} n^{\alpha}\right)}
$$

where

$$
\begin{aligned}
L_{e f f}^{\prime}= & \dot{q}^{s} p_{s}+n^{\alpha} \Phi_{\alpha}-i \bar{c}_{\beta}\left(\frac{\partial g^{\beta}}{\partial n^{\alpha}} \partial_{t}-\frac{\partial g^{\beta}}{\partial n^{\gamma}} C_{\alpha \lambda}^{\gamma} n^{\lambda}+\left\{\Phi_{\alpha}, f^{\beta}\right\}\right) c^{\alpha} \\
& -\bar{c}_{\xi} \bar{c}_{\eta}\left(\frac{\partial g^{\eta}}{\partial n^{\delta}}\left\{f^{\xi}, C_{\alpha \beta}^{\delta}\right\}+3 \frac{\partial g^{\xi}}{\partial n^{\delta}} \frac{\partial g^{\eta}}{\partial n^{\lambda}} U_{\alpha \beta \gamma}^{(2) \delta \lambda} n^{\gamma}\right) c^{\alpha} c^{\beta} \\
& -i \bar{c}_{\alpha} \bar{c}_{\xi} \bar{c}_{\eta} \frac{\partial g^{\xi}}{\partial n^{\lambda}} \frac{\partial g^{\eta}}{\partial n^{\sigma}}\left\{f^{\alpha}, U_{\beta \gamma \delta}^{(2) \lambda \sigma}\right\} c^{\beta} c^{\gamma} c^{\delta}
\end{aligned}
$$

and $q^{s}=\left(\eta_{i}^{a}, \omega^{a}\right), p_{s}=\left(\widetilde{E}_{a}^{i}, \chi_{a}\right)$.

This completes construction of the path integral for the Hilbert-Palatini gravity.One can see that dependence of structure constants on canonical variables leads to appearance of multighost interaction terms in (41). By an appropriate choice of gauge fixing functions one can eliminate these terms. All nonvanishing components of $U^{(2)}$ have upper indices corresponding to the Gauss or Lorentz constraints. Therefore, if the functions $g^{\alpha}$ do not depend on the Lagrange multipliers $\mathcal{N}_{G}$ and $\mathcal{N}_{L}$, all terms with $U^{(2)}$ disappear. If, furthermore, the functions $f^{\alpha}$ do not depend on canonical coordinates $q^{s}$, the Poisson bracket $\left\{f^{\xi}, C_{\alpha \beta}^{\delta}\right\}$ vanishes and the remaining higher ghost terms disappear also. In such a case, general structure of the path integral is identical to that of rank one Yang-Mills theory. For short, these gauges will be called the Yang-Mills (YM) gauges. They play an important role in path integral quantization of the Ashtekar gravity.

\section{CONSTRAINTS VERSUS REALITY CONDITIONS}

In this section we establish relation between solutions of the constraints in the real Hilbert-Palatini formulation and the reality conditions (8) and (9) of the Ashtekar gravity. Let us recall expressions for the complex canonical variables $P$ and $A$ in terms of the real canonical variables:

$$
\begin{aligned}
P_{a}^{i} & =i\left(\widetilde{E}_{a}^{i}-i \varepsilon_{a}{ }^{b c} \widetilde{E}_{b}^{i} \chi_{c}\right), \\
A_{j}^{a} & =\xi_{j}^{a}-i\left(\eta_{j}^{a}+\varepsilon^{a b c} \xi_{j}^{b} \chi_{c}\right), \\
\xi_{j}^{a} & =r_{j}^{a}-\frac{1}{2} \varepsilon^{a b c} \omega_{b}{\underset{\sim}{j}}_{j}^{c},
\end{aligned}
$$

$r_{j}^{a}$ is given by the equation (28).

Here it will be demonstrated the reality conditions (8) and (9) are satisfied by (42) provided the canonical variables of the real theory satisfy the Gauss law and the Lorentz constraint. Moreover, we shall prove that the Ashtekar action is real under the same conditions. The last statement is not completely trivial even though real Hilbert-Palatini action is related to complex Ashtekar action by a canonical transformation. The point is that this transformation is not canonical on the whole phase space [1]. Thus for our basis in the phase space reality of the Ashtekar action must be checked independently. 
The first reality condition (8) is satisfied trivially. Let us rewrite (9) in a more explicit form. Time evolution $P_{a}^{l} P_{a}^{j}$ is given by Poisson bracket of total complex Hamiltonian (7) and $P_{a}^{P} P_{a}^{j}$ :

$$
\begin{aligned}
\partial_{t}\left(P_{a}^{l} P_{a}^{j}\right)= & \left\{\int d t d^{3} x\left(A_{0}^{a} \mathcal{G}_{a}+\mathcal{N}_{D}{ }^{i} H_{i}+\underset{\sim}{\mathcal{N}} H\right), P_{a}^{l} P_{a}^{j}\right\}_{C} \\
= & -2\left(2 P_{a}^{l} P_{a}^{j} \partial_{i} \mathcal{N}_{D}{ }^{i}-P_{a}^{k} P_{a}^{l} \partial_{k} \mathcal{N}_{D}^{j}-P_{a}^{k} P_{a}^{j} \partial_{k} \mathcal{N}_{D}{ }^{l}+\mathcal{N}_{D}{ }^{i} \partial_{i}\left(P_{a}^{l} P_{a}^{j}\right)\right) \\
& +2\left(\nabla_{k} P_{a}^{k}\right)\left(\mathcal{N}_{D}^{j} P_{a}^{l}+\mathcal{N}_{D}^{l} P_{a}^{j}\right) \\
& -2 \mathcal{\sim} \varepsilon^{a b c} P_{a}^{i}\left(P_{c}^{j} \nabla_{i} P_{b}^{l}+P_{c}^{l} \nabla_{i} P_{b}^{j}\right) .
\end{aligned}
$$

First line of (43) is real for real $\mathcal{N}_{D}{ }^{i}$ due to the first reality condition (8). Second line disappears due to the Gauss law constraint. Therefore, to ensure real metric evolution one must require

$$
\operatorname{Im}\left(\varepsilon^{a b c} P_{a}^{i}\left(P_{c}^{j} \nabla_{i} P_{b}^{l}+P_{c}^{l} \nabla_{i} P_{b}^{j}\right)\right)=0 .
$$

The condition (44) can be presented as $\operatorname{Im}\left\{P_{a}^{l} P_{a}^{j}, H\right\}_{C}=0$. It is clear that this condition is invariant under complex $S O(3)$ transformations. These transformations can be used to put $\chi=0$. One can easily demonstrate that for the fields (42) the condition (44) is satisfied.

Now let us prove that under the same conditions

$$
\operatorname{Im} H_{i}=\operatorname{Im}\left(H_{i}+2 A_{i}^{a} \mathcal{G}_{a}\right)=0 .
$$

From the equations (11) and (34) one can see that $\{\mathcal{G}, \mathcal{G}\}_{C} \sim \mathcal{G}$ and $\left\{\Phi^{D}, \mathcal{G}\right\} \sim \mathcal{G}$. Hence the surface $\mathcal{G}=0$ is invariant under complex $S O(3)$ transformations and real diffeomorphisms. Since $\left\{\mathcal{G}, H_{i}+2 A_{i}^{a} \mathcal{G}_{a}\right\}_{C} \sim \mathcal{G}$ and $\left\{\Phi^{D}, \operatorname{Im}\left(H_{i}+2 A_{i}^{a} \mathcal{G}_{a}\right)\right\} \sim \operatorname{Im}\left(H_{i}+2 A_{i}^{a} \mathcal{G}_{a}\right)$, these transformations map solutions of (45) to themselves inside the surface $\mathcal{G}=0$. One can use $S O(3)$ transformations and diffeomorphisms to impose the condition $\chi=0$ everywhere, and $\partial_{k} \widetilde{E}_{a}^{j}=0$ at a certain point. At this point one must only check cancellation of second derivatives of $\widetilde{E}$. This is straightforward to do by using the equations (12), (28) and the explicit form (23) of the constraint $\mathcal{G}=\Phi^{G}+i \Phi^{L}$.

To prove that $\operatorname{Im} H=0$ one can use the Lorentz boosts to put $\chi=0$. This makes the calculations quite elementary even without further gauge fixing.

By straightforward calculations one can demonstrate that imaginary part of the kinetic term $P_{a}^{j} \partial_{t} A_{j}^{a}$ is a total derivative and thus can be discarded in quantization. This is done in the Appendix C.

As it was advertised at the beginning of this section, we demonstrated that the complex canonical variables satisfy the reality conditions on the surface of the equations (42), the second class constraint (27) and two first class constraints $\Phi^{G}$ and $\Phi^{L}$. Note, that the reality conditions admit more solutions. For example, one can interchange real and imaginary parts of $P_{a}^{j}$.

\section{PATH INTEGRAL QUANTIZATION OF THE ASHTEKAR GRAVITY}

In this section we derive a path integral for the Ashtekar gravity from the one for the Hilbert-Palatini gravity. Consider functional (40) in an YM-gauge.

$$
\begin{array}{r}
Z[j, J]=\int \mathcal{D} \eta_{i}^{a} \mathcal{D} \tilde{E}_{a}^{i} \mathcal{D} \omega^{a} \mathcal{D} \chi_{a} \\
\mathcal{D} \mathcal{N}_{G} \mathcal{D} \mathcal{N}_{L} \mathcal{D} \mathcal{N}_{D}^{i} \mathcal{D} \mathcal{\sim}_{\sim} \mathcal{D} c^{\alpha} \mathcal{D} \bar{c}_{\alpha} \delta\left(f^{\alpha}+g^{\alpha}\right) \\
\times \exp \left(i \int d t\left(L_{e f f}^{\prime}+j_{a}^{i} \eta_{i}^{a}+J_{i}^{a} \widetilde{E}_{a}^{i}\right)\right)
\end{array}
$$

We dropp out the sources for the Lagrange multipliers, $\chi$ and $\omega$. Discussion of the source terms is postponed to the end of this section.

Since the gauge fixing functions $g^{\alpha}$ does not depend on the Gauss and Lorentz Lagrange multipliers, integration over these Lagrange multipliers gives $\delta$-functions of the corresponding constraints, $\delta\left(\Phi_{a}^{G}\right) \delta\left(\Phi_{a}^{L}\right)$. This means that in fact we are working on the surface of these constraints. In the previous section it is shown that on this surface imaginary part of the Ashtekar action vanishes. Thus one can write

$$
L_{e f f}^{\prime}=L_{A}(P, A)-i \bar{c}_{\beta}\left(\frac{\partial g^{\beta}}{\partial n^{\alpha}} \partial_{t}-\frac{\partial g^{\beta}}{\partial n^{\gamma}} C_{\alpha \lambda}^{\gamma} n^{\lambda}+\left\{\Phi_{\alpha}, f^{\beta}\right\}\right) c^{\alpha}
$$


We assume that complex canonical variables are expressed in terms of real canonical variables by means of (42).

One can integrate over $\omega^{a}$ by using the delta function of the Lorentz constraint $\Phi^{L}$. This is equivalent in effect to the substitution:

$$
\omega^{a}(\widetilde{E}, \eta, \chi):=\left(\delta_{a b}+\frac{\chi_{a} \chi_{b}}{1-\chi^{2}}\right) \partial_{i} \widetilde{E}_{b}^{i}+\widetilde{E}_{a}^{i} \eta_{i}^{b} \chi_{b}-\frac{\chi_{a}}{1-\chi^{2}}\left(\widetilde{E}_{b}^{i} \eta_{i}^{b}-\widetilde{E}_{b}^{i} \chi_{b} \eta_{i}^{c} \chi_{c}\right)
$$

The path integral measure is multiplied by

$$
\Delta_{1}=\operatorname{det}^{-1}\left(\delta_{a b}-\chi_{a} \chi_{b}\right)=\prod_{x, t} \frac{1}{1-\chi^{2}} .
$$

Now we are ready to change the integration variables in 46):

$$
\begin{aligned}
\tilde{E}_{a}^{i} \longrightarrow P_{a}^{i} & =i \widetilde{E}_{a}^{i}+\varepsilon^{a b c} \tilde{E}_{b}^{i} \chi_{c} \\
\eta_{i}^{a} \longrightarrow A_{i}^{a} & =\xi_{i}^{a}-i\left(\eta_{i}^{a}+\varepsilon^{a b c} \xi_{i}^{b} \chi_{c}\right)
\end{aligned}
$$

This gives rise to a determinant

$$
\begin{aligned}
& \Delta_{2}=\operatorname{det}^{-1}\left(1 i \delta_{j}^{i} \delta_{a}^{b}+\delta_{j}^{i} \varepsilon^{a b c} \chi_{c}\right) \operatorname{det}^{-1}\left(\frac { 1 } { 2 ( 1 - \chi ^ { 2 } ) } \left(-2 \delta_{i}^{j} \varepsilon^{a b c} \chi_{c}+\varepsilon^{a p q} \widetilde{E}_{q}^{j}\left(\delta_{p b}-\chi_{p} \chi_{b}\right){\underset{\sim}{i}}_{i}^{d} \chi_{d}\right.\right. \\
& \left.-\chi_{a} \varepsilon^{d p q} \underset{\sim}{E_{i}^{d}} \tilde{E}_{q}^{j}\left(\delta_{p b}-\chi_{p} \chi_{b}\right)\right)-i\left(\delta_{i}^{j} \delta_{b}^{a}+\frac{1}{2\left(1-\chi^{2}\right)}\left(2 \delta_{i}^{j}\left(\delta_{b}^{a} \chi^{2}-\chi_{a} \chi_{b}\right)\right.\right.
\end{aligned}
$$

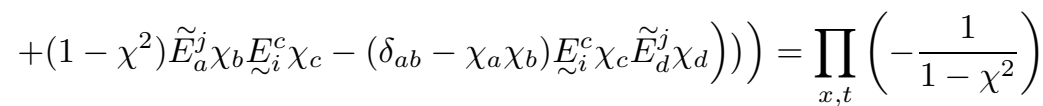

Note, that if all the gauge fixing functions $f$ depend on the real fields $\chi$ and $\widetilde{E}$ through $P$ only, the ghost action becomes degenerate (see (34)). This is a manifestation of the fact that the Lorentz constraint is "superfluous" in the complex Ashtekar gravity. Therefore, we must fix corresponding gauge freedom by means of a condition on $\chi$ :

$$
\chi^{a}=\chi_{(0)}^{a}(\tilde{E})
$$

where $\chi_{(0)}$ is a given function.

Before integrating over $\chi$ let us rewrite (52) in a different form. By inverting the first equation in (42), one obtains

$$
\tilde{E}_{a}^{i}=\left(\frac{\varepsilon^{a b c} \chi_{c}}{1-\chi^{2}}-i \frac{\delta_{a b}-\chi_{a} \chi_{b}}{1-\chi^{2}}\right) P_{b}^{i}=\pi_{a}^{b}(\chi) P_{b}^{i} .
$$

Due to 52 one can replace $\chi$ by $\chi_{(0)}(\tilde{E})$. Right hand side of (53) becomes $\tilde{E}$ dependent. This dependence, however, can be removed at least locally be means of formal power series expansion. As a result, we obtain

$$
\widetilde{E}_{a}^{i}=\bar{\pi}_{a}^{b}(P) P_{b}^{i}
$$

where $\bar{\pi}$ is a function of $P$ but not of $P^{*}$, which depends on a choice of the gauge fixing function $\chi^{(0)}$. For the present analysis explicit form of $\bar{\pi}$ is of no importance. Note, that simple relation $\widetilde{E}=\operatorname{Im} P$ would not work, because it depends both on $P$ and its complex conjugate.

One can replace (52) by the condition

$$
\chi=\chi_{(0)}(\bar{\pi} P)=\bar{\chi}(P)
$$

The two conditions (52) and (55) are equivalent since they select the same surfaces in the phase space. However, ghost terms and Jacobian factors appearing due to delta functions of gauge conditions are different for (52) and (55). In the final result these differences compensate each other, as one can easily show using geometric interpretation of the Faddeev-Popov determinant.

Let us integrate over $\chi$ with the help of the delta function $\delta(\chi-\bar{\chi}(P))$. Since we already changed variables to $P$ and $A$, no Jacobian factor appears.

Intergation over $P$ and $A$ should be understood as a contour integration in complex space. One integrates along the lines defined by the reality conditions and the equations (52) and (48). As usual, there are real parameters which label points of the contours in the complex planes. These are $E$ and $\eta$. Since the fields $\omega$ and $\chi$ are already excluded, we do not integrate over position of the contours. 
Consider the ghost action. Integration over $\bar{c}$ and $c$ gives the following functional determinant:

$$
\operatorname{det}\left(\frac{\partial g^{\beta}}{\partial n^{\alpha}} \partial_{t}-\frac{\partial g^{\beta}}{\partial n^{\gamma}} C_{\alpha \lambda}^{\gamma} n^{\lambda}+\left\{\Phi_{\alpha}, f^{\beta}\right\}\right)
$$

Let us separate indices corresponding to the Lorentz boosts: $\left\{\Phi_{\alpha}\right\}=\left\{\Phi_{a}^{L} ; \Phi_{\mu}\right\},\left\{f^{\alpha}\right\}=\left\{\chi^{a}-\bar{\chi}^{a}(P) ; f^{\mu}(\chi, P)\right\}$, $\left\{g^{\alpha}\right\}=\left\{0 ; g^{\mu}\right\}$. Greek indices from the middle of the alphabet correspond to the Gauss law, diffeomorphism and Hamiltonian constraints. Matrix elements in (56) contain the following brackets:

$$
\begin{aligned}
& \left\{\Phi_{\mu}, f^{\nu}(\chi, P)\right\}=\left\{\Phi_{\mu}, P\right\} \frac{\delta f^{\nu}}{\delta P}+\frac{\delta \Phi_{\mu}}{\delta \omega} \frac{\delta f^{\nu}}{\delta \chi} \\
& \left\{\Phi_{\mu}, \chi-\bar{\chi}(P)\right\}=\frac{\delta \Phi_{\mu}}{\delta \omega}-\left\{\Phi_{\mu}, P\right\} \frac{\delta \bar{\chi}}{\delta P}
\end{aligned}
$$

where summation indices are suppressed. Let us multiply the lines corresponding to $\chi^{a}-\bar{\chi}^{a}$ by $-\delta f^{\nu} / \delta \chi^{a}$ and add them to the $f^{\nu}$ lines. This produces the matrix elements:

$$
\begin{aligned}
& \frac{\partial g^{\nu}}{\partial n^{\mu}} \partial_{t}-\frac{\partial g^{\nu}}{\partial n^{\rho}} C_{\mu \sigma}^{\rho} n^{\sigma}+\left\{\Phi_{\mu}, P\right\}\left(\frac{\delta f^{\nu}}{\delta P}+\frac{\delta f^{\nu}}{\delta \chi} \frac{\delta \bar{\chi}}{\delta P}\right)= \\
& \frac{\partial g^{\nu}}{\partial n^{\mu}} \partial_{t}-\frac{\partial g^{\nu}}{\partial n^{\rho}} C_{\mu \sigma}^{\rho} n^{\sigma}+\left\{\Phi_{\mu}^{[C]}, f^{\nu}(\bar{\chi}(P), P)\right\}_{C} .
\end{aligned}
$$

$\Phi_{\mu}^{[C]}$ is the Ashtekar constraint corresponding to $\Phi_{\mu}, \operatorname{Re} \Phi_{\mu}^{[C]}=\Phi_{\mu}$. In the last line we used that $\left\{\Phi_{\mu}, P\right\}=\left\{\Phi_{\mu}^{[C]}, P\right\}_{C}$ due to (34). The equation (58) means that one replace $\chi$ by $\bar{\chi}$ in the gauge fixing functions $f^{\nu}$.

Consider the two columns in (56) corresponding to the Gauss law and Lorentz constraints. Due to (34) $\left\{\Phi^{G}, f(P)\right\}=$ $i\left\{\Phi^{L}, f(P)\right\}$. Therefore, by multiplying the column with $\Phi^{G}$ by $-i$ and adding it to the column with $\Phi^{G}$ one obtains zeros almost everywhere, except for the lines corresponding to the gauge conditions $\chi^{a}-\bar{\chi}^{a}(P)$. As a result, one can represent the determinant (56) as a product of two determinants:

$$
\Delta_{3} \operatorname{det}\left(\frac{\partial g^{\nu}}{\partial n^{\mu}} \partial_{t}-\frac{\partial g^{\nu}}{\partial n^{\rho}} C_{\mu \sigma}^{\rho} n^{\sigma}+\left\{\Phi_{\mu}^{[C]}, f^{\nu}(\bar{\chi}(P), P)\right\}_{C}\right)
$$

where

$$
\Delta_{3}=\operatorname{det}\left\{\Phi_{a}^{L}-i \Phi_{a}^{G}, \chi^{b}\right\}=\operatorname{det}\left(\left(\delta_{a b}-\chi_{a} \chi_{b}\right)+i \varepsilon^{a b c} \chi_{c}\right)=\prod_{x, t}\left(1-\chi^{2}\right)^{2}
$$

From the expressions (49), (51) and (60) one can see that all $\Delta$ 's cancel each other up to an overall minus sign which can be absorbed in reversed orientation of the contour of the $A$-integration. The path integral is now rewritten in terms of the Ashtekar variables:

$$
Z[\bar{j}, \bar{J}]=\int_{R} \mathcal{D} A_{i}^{a} \mathcal{D} P_{a}^{i} \mathcal{D} \mathcal{N}_{D}^{i} \mathcal{D} \mathcal{N} \mathcal{D} A_{0}^{a} \mathcal{D} c^{\mu} \mathcal{D} \bar{c}_{\mu} \delta\left(f^{\mu}+g^{\mu}\right) e^{i \int d t\left(L_{\text {eff }}^{\prime}+\bar{j}_{a}^{i} A_{i}^{a}+\bar{J}_{i}^{a} P_{a}^{i}\right)}
$$

where

$$
L_{e f f}^{\prime}=L_{A}-i \bar{c}_{\nu}\left(\frac{\partial g^{\nu}}{\partial n^{\mu}} \partial_{t}-\frac{\partial g^{\nu}}{\partial n^{\rho}} C_{\mu \sigma}^{\rho} n^{\sigma}+\left\{\Phi_{\mu}^{[C]}, f^{\nu}(\bar{\chi}(P), P)\right\}_{C}\right) c^{\mu}
$$

The subscript $R$ means contour integration in complex spaces along lines defined by the reality conditions. Integration over $\mathcal{N}_{L}$ (which is essentially an imaginary part of $A_{0}$ ) has been already performed to produce a delta function of the Lorentz constraint. This delta function, in turn, has been used to integrate over $\omega$. Thus in (61) we integrate over real part of $A_{0}$. This integral gives $\delta\left(\Phi^{G}\right)=\delta(\mathcal{G})$. The equation $\mathcal{G}=0$ can be considered as a complex equation because $\operatorname{Im} \mathcal{G}=0$ is supplied by the reality conditions. The same is true for the gauge conditions $f^{\mu}+g^{\mu}=0$. A fascinating property of these complex delta functions is possibility to integrate over complex variables without explicit transition to real coordinates on a contour.

By comparing (11) and (32), one can see that $C_{\mu \sigma}^{\rho}$ are just structure constants of the Ashtekar gravity (Note, that this property does not hold in the variables used by Henneaux [8]) Therefore, the ghost term in (61) produces the ordinary Faddeev-Popov determinant for the Ashtekar gravity. The path integral (61) coincides with what one would write naively just ignoring any Jacobian factors which may arise from the reality conditions and fixing the Lorentz 
gauge freedom. Some remarks are in order. First of all, the result (61) is valid for a certain class of gauges only. We are not allowed to impose gauge condition on $A_{0}^{a}$. This restriction is needed (i) to cancel contributions to the path integral of the second order structure functions (which are zero for the Ashtekar gravity [6]), and (ii) to ensure delta functions of the complex Gauss law constraint. While (i) seems to depend on a particular choice of basic variables and constraints because rank of and algebra is not an invariant, the second point (ii) looks more fundamental. The complex Gauss law constraint is needed to prove vanishing of imaginary part of the Ashtekar action. We are not allowed to impose gauge conditions on the connection variables. The ultimate reason for this is that the last line of (34) is not true if we replace $P$ by $A$. This restriction will receive a natural explanation in the next section in a framework of the Faddeev path integral. In all other respects the gauge conditions $f^{\alpha}+g^{\alpha}$ are arbitrary. For a given set of admissible YM gauges one can first express $\chi^{a}$ from three of them and then denote the remaining gauge conditions by $f^{\mu}+g^{\mu}$.

Path integral for the Ashtekar gravity was previously considered by the present authors and I. Grigentch in the one-loop approximation over de Sitter background [21] and for the Bianchi IX finite dimensional model [22]. In these simple cases the reality conditions do not lead to any Jacobian factors if one uses gauge conditions of the YM type. We observed also that one runs into troubles if gauge conditions are imposed on the connection variables.

Using of this or that gauge condition is just a matter of convenience. In principle, it is enough to formulate the path integral in just one gauge. All physical results are to be gauge independent. However, extension of our results for arbitrary gauge conditions still poses an interesting problem from both technical and aesthetic points of view.

Note, that we excluded sources for $\chi, \omega$ and Lagrange multipliers. Sources for $\chi$ and $\omega$ are not needed because in the present formulation these fields are absent. Moreover, $\chi$ and $\omega$ can be considered as composite fields. Sources

for $\underset{\sim}{N}$ and $\mathcal{N}_{D}$ can be easily restored without any modification in our procedure. Therefore, we have enough sources to describe any Green functions of the four-metrics and three-dimensional connections. If, however, we introduce a source for $A_{0}^{a}$, it penetrates into the delta functions of the Gauss law and Lorentz constraints and destroys reality of the Ashtekar action. Green functions of $A_{0}$ are not defined in our approach. At the last step we introduced sources $\bar{J}$ and $\bar{j}$ for $P$ and $A$. This makes exponential in (61) complex. Thus, strictly speaking, the path integral is not well defined, even though all finite order Green functions do exist. If one wishes to be on a safe side, one can easily return to the original sources $J$ and $j$ for $\widetilde{E}$ and $\eta$.

\section{THE FADDEEV PATH INTEGRAL}

In this section we give a more simple derivation of the Faddeev path integral [23] for the Ashtekar gravity, which does not rely upon heavy machinery of the BRST quantization. This also seems to be a proper place to discuss triad form of the reality conditions. For a dynamical system with canonical variables $q^{s}, p_{s}$, first class constraints $\Phi_{a}$ and weakly vanishing Hamiltonian, such as the Hilbert-Palatini gravity, the Faddeev path integral reads:

$$
Z=\int \mathcal{D} q \mathcal{D} p \mathcal{D} n F \delta\left(f^{\alpha}\right) \exp \left(i \int d t\left(\dot{q}^{s} p_{s}+n^{\alpha} \Phi_{\alpha}\right)\right)
$$

where $f^{\alpha}$ are gauge fixing functions of the dynamical variables. $F$ is the Faddeev-Popov determinant, $F=$ $\operatorname{det}\left\{\Phi_{\alpha}, f^{\beta}\right\}$. We do not show the source terms explicitly. The expression (63) can be obtained by from the path integral (40) by choosing $g^{\alpha}=0$ and integrating over the ghost fields $c$ and $\bar{c}$. Of course, starting point of the original derivation 23] of the Faddeev path integral was not the BRST approach.

To make the presentation as simple as possible, we fix Lorentz boosts by the condition

$$
\chi=0 .
$$

Now we integrate over $\mathcal{N}_{L}^{a}, \chi$ and $\omega$. Again, integration over $\omega$ is equivalent to the following substitution:

$$
\omega_{a}:=\partial_{j} \widetilde{E}_{a}^{j}
$$

If the remaining gauge fixing conditions $f^{\mu}$ are functions of $\widetilde{E}$ only, the Poisson brackets $\left\{f^{\mu}, \Phi_{L}^{a}\right\}$ vanish on the surface (64). Hence the Faddeev-Popov determinant takes the form

$$
F=\operatorname{det}\left\{f^{\mu}(\widetilde{E}), \Phi_{\nu}\right\}=\operatorname{det}\left\{f^{\mu}(-i P), \Phi_{\nu}^{[C]}\right\}_{C}
$$

The gauge (64) means that we are using reality conditions in the triad form 


$$
\operatorname{Re} P_{a}^{i}=0, \quad \operatorname{Re}\left(\partial_{t} P_{a}^{i}\right)=0
$$

instead of the metric reality conditions (8) and (9).

The change of variables $(\widetilde{E}, \eta) \rightarrow(P, A)$ gives unit Jacobian factor. Our prove of vanishing of imaginary part of the Ashtekar action is still valid. Hence we arrive at the path integral for the Ashtekar gravity in the Faddeev form:

$$
Z=\int_{R} \mathcal{D} P \mathcal{D} A \mathcal{D} \underset{\sim}{\sim} \mathcal{D} \mathcal{N}_{D} \mathcal{D} A_{0} F \delta\left(f^{\mu}(-i P)\right) \exp \left(i S_{A}\right)
$$

where subscript $R$ means now that the contour of integration is defined by the reality conditions (67). Of course, most of the comments of the previous section apply here also.

\section{DISCUSSION}

Main result of the present paper is the path integral (61) for the Ashtekar gravity, which is a kind of contour integral. As a byproduct, we also constructed the BRST quantization of the Hilbert-Palatini gravity. Main features of our approach were discussed in detail in the section VI. Here we speculate on perspectives of this approach.

The path integral (61) is obtained with certain restrictions on possible gauge conditions. In principle, one can transform (61) to any other gauge by means of the Faddeev-Popov trick [7]. However, this trick is not so easy to implement in the present context due to reality conditions and quite unusual rules of the functional integration. Perhaps restrictions on the gauge conditions may be weakened or even lifted altogether. Anyhow, one should formulate criteria of admissibility of gauge conditions for the Ashtekar gravity in terms of the Ashtekar variables without referring to the Hilbert-Palatini gravity. This definitely will not be easy to do. In general, a function of $P$ is complex valued. Therefore, a condition $f=0$ implies two real gauge fixing conditions $\operatorname{Re} f=0$ and $\operatorname{Im} f=0$ even if reality conditions are taken into account. Even the requirement that a given set of gauge conditions removes correct number of degrees of freedom looks quite non-trivial. One may hope to overcome these difficulties by using the generalized Wick rotation [24.

We must admit that for degenerate triad our analysis is incomplete. This reflects a well known problem of the Ashtekar gravity which exists already at the classical level.

An intriguing feature of (61) is that it is a contour integral. The contour of integration can be deformed as far as the reality conditions allow (This corresponds to arbitrariness of gauge fixing in the Hilbert-Palatini action.) One may hope, that certain deformations are possible even beyond these limits. If this is really so, some interesting properties of quantum gravity can manifest themselves.

\section{ACKNOWLEDGMENTS}

This work was supported by the Russian Foundation for Fundamental Research, grant 97-01-01186, and by GRACENAS through grant 97-0-14.1-61 (D.V.) and Young Investigator Program (S.A.).

\section{APPENDIX A:}

Let us solve the second class constraint (27). The matrix $\mathcal{A}_{A B}$ is defined by the $r^{2}$ terms in the Hamiltonian constraint $\Phi^{H}$. We have:

$$
\lambda^{A} \mathcal{A}_{A B} \lambda^{B}=r_{b}^{c} \mathcal{A}_{b b^{\prime}}^{c c^{\prime}} r_{b^{\prime}}^{c^{\prime}}
$$

where $r_{b}^{c}=r_{j}^{c} \tilde{E}_{b}^{j}$. We can identify non-dynamical components of the connection $\lambda_{A}$ with the symmetric matrices $r_{a}^{b}$. The operator

$$
\mathcal{A}_{b b^{\prime}}^{c c^{\prime}}=\left(1-\chi^{2}\right) \varepsilon^{a b b^{\prime}} \varepsilon^{d c c^{\prime}} X_{a d}, \quad X_{a d}=\delta_{a d}-\chi_{a} \chi_{d}
$$

acts on the space of symmetric $3 \times 3$ matrices. One can represent it in the following form:

$$
\mathcal{A}_{b b^{\prime}}^{c c^{\prime}}=\left(1-\chi^{2}\right)^{2}\left(X^{b c} X^{b^{\prime} c^{\prime}}-X^{b c^{\prime}} X^{c b^{\prime}}\right)
$$


where $X^{b c}$ is inverse of $X_{b c}$. Inverse of (A3) is easily found to be

$$
\left(\mathcal{A}^{-1}\right)_{b b^{\prime}}^{c c^{\prime}}=\left(1-\chi^{2}\right)^{-2}\left(\frac{1}{2} X_{b c} X_{b^{\prime} c^{\prime}}-X_{b c^{\prime}} X_{c b^{\prime}}\right)
$$

Linear part of the Hamiltonian constraint reads:

$$
\mathcal{B}_{A} \lambda^{A}=\varepsilon^{a b c} \widetilde{E}_{b}^{i}\left(\widetilde{E}_{c}^{j} r_{d}^{a}\left(\partial_{i}{\underset{\sim}{j}}_{j}^{d}\right)\left(1-\chi^{2}\right)+r_{c}^{d} \chi_{d} \partial_{i} \chi_{a}-\left(1-\chi^{2}\right) \chi_{a} r_{c}^{d} \eta_{i}^{d}+X_{a g} \eta_{i}^{g} r_{c}^{d} \chi_{d}\right)=\mathcal{B}_{a}^{b} r_{b}^{a}
$$

Note, that (A5) does not contain derivatives of $r_{a}^{b}$. Hence the second class constraint (27) can be solved for $r_{a}^{b}$ :

$$
r_{d}^{c}=\frac{1}{2}\left[\left(\mathcal{A}^{-1}\right)_{d b}^{c a}+\left(\mathcal{A}^{-1}\right)_{d a}^{c b}\right] \mathcal{B}_{a}^{b}
$$

Substitution of (A4) and(A5) in (A6) gives the expression (28). The Hamiltonian constraint takes the form $\Phi^{H}=$ $\Phi_{0}^{H}+\mathcal{B}_{a}^{b}\left(\mathcal{A}^{-1}\right)_{b d}^{a c} \mathcal{B}_{c}^{d}$, which is written explicitly in (29).

\section{APPENDIX B:}

In this Appendix we define structure functions $U^{(n)}$ of the Hilbert-Palatini gravity. For $n=0$ and $n=1$ they are

$$
U_{\alpha}^{(0)}=\Phi_{\alpha}, \quad U_{\alpha \beta}^{(1) \gamma}=-\frac{1}{2} C_{\alpha \beta}^{\gamma},
$$

with $C_{\alpha \beta}^{\gamma}$ defined by the algebra (32) through the relation $\left\{\Phi_{\alpha}, \Phi_{\beta}\right\}=C_{\alpha \beta}^{\gamma} \Phi_{\gamma}$. Higher order structure functions are defined through repeated Poisson brackets of the constraints

$$
2 U_{\alpha \beta \gamma}^{(2) \xi \eta} \Phi_{\eta}=D_{\alpha \beta \gamma}^{(1) \xi}=\frac{1}{2}\left(\left\{\Phi_{\alpha}, C_{\beta \gamma}^{\xi}\right\}-C_{\beta \gamma}^{\delta} C_{\alpha \delta}^{\xi}\right)_{[\alpha \beta \gamma]}
$$

where $\left[\alpha_{1} \cdots \alpha_{n}\right]$ means antisymmetrization in $\alpha_{1} \cdots \alpha_{n}$ with the weight $1 / n$ !. In actual calculations it is convenient to replace antisymmetrization by multiplication by anticommuting ghosts. The indices $\alpha, \beta, \ldots$ denote constraints at different coordinate points. Therefore, antisymmetrisation over coinciding indices does not necessarily give zero.

If less than two indices among $\alpha, \beta$ and $\gamma$ correspond to the Hamiltonian constraint, the structure functions $C$ in (B2) become field independent structure constants, and the second order structure functions $U_{\alpha \beta \gamma}^{(2) \xi \eta}$ vanish by virtue of ordinary Bianchi identities. Hence, one must calculate only the structure functions with a pair of indices, say $\beta$ and $\gamma$, corresponding to the Hamiltonian constraint. From now on, an index representing the Hamiltonian constraint will be denoted by $0, \Phi^{H} \equiv \Phi_{0}$. We put $\gamma=\beta=0$.

It is convenient to introduce a connection field of the Lorentz group $S O(3,1): A_{i}^{p}=\left(\xi_{i}^{a}, \eta_{i}^{x}+\varepsilon^{x g f} \xi_{i}^{g} \chi_{f}\right), p=1, \ldots, 6$. $f_{p q}^{r}$ will denote structure constants of corresponding Lie algebra.

From (32) it is clear that the canonical momenta enter the first order structure functions $C$ through the vector $K^{j}[n, m]=\left(n \partial_{i} m-\partial_{i} n m\right) K^{i j}$, where $K^{i j}$ is defined in (33). Later $n$ and $m$ will be replaced by ghost fields. Thus an order is essential. $n$ always precedes $m$. The tensor $K$ has the following Poisson brackets with the constraints:

$$
\begin{aligned}
& \left\{\Phi_{a}^{G}, K^{i j}\right\}=\left\{\Phi_{a}^{L}, K^{i j}\right\}=0, \quad\left\{c^{0} \Phi_{0}, K^{j}\left[c^{0}, c^{0}\right]\right\}=0, \\
& \left\{c^{k} \Phi_{k}^{D}, K^{i j}\right\}=2\left(2 K^{i j} \partial_{k} c^{k}+c^{k} \partial_{k} K^{i j}-\partial_{k} c^{j} K^{i k}-\partial_{k} c^{i} K^{k j}\right)
\end{aligned}
$$

where contraction with anticommuting ghosts $c$ is used for antisymmetrization in corresponding indices.

Let us calculate $c^{\alpha} D_{\alpha 00^{\prime}}^{(1) \xi} c^{0}(x) c^{0}\left(x^{\prime}\right)$. Consider various cases for $\alpha$. If $\Phi_{\alpha}=\Phi_{p}=\left(\Phi^{G}, \Phi^{L}\right)$ and $\Phi_{\xi}=\Phi_{0}\left(=\Phi^{H}\right)$ or $\Phi_{\xi}=\Phi^{D}$ this quantity vanishes due to (B3). For $\Phi_{\xi}=\Phi_{q}$ one obtains

$$
c^{p} D_{p 00^{\prime}}^{(1) q} c^{0}(x) c^{0}\left(x^{\prime}\right)=\frac{2}{3} K^{j}\left[c_{0}(x), c_{0}\left(x^{\prime}\right)\right]\left(-\left\{c^{p} \Phi_{p}, A_{j}^{q}\right\}+f_{r p}^{q} A_{j}^{r} c^{p}+\partial_{j} c^{q}\right) \delta\left(x, x^{\prime}\right)
$$

As a part of our summation convention we assume integration over all continuous coordinates here and in the equations bellow. The expression (B4) is zero due to (31). This implies that $U_{00 p}^{(2) \xi \eta}=0$.

Let us put $\Phi_{\alpha}=\Phi_{i}^{D}$. We are to evaluate:

$$
c^{i} D_{i 0^{\prime} 0^{\prime \prime}}^{(1) \xi} c^{0}\left(x^{\prime}\right) c^{0}\left(x^{\prime \prime}\right)=\frac{1}{6} c^{i}\left(\left\{\Phi_{i}, C_{0^{\prime} 0^{\prime \prime}}^{\xi}\right\}-C_{0^{\prime} 0^{\prime \prime}}^{\beta} C_{i \beta}^{\xi}-2 C_{i 0^{\prime}}^{\beta} C_{0^{\prime \prime} \beta}^{\xi}\right) c^{0}\left(x^{\prime}\right) c^{0}\left(x^{\prime \prime}\right) .
$$


First we observe that the only non-vanishing function $C$ with zero upper index is $C_{o i}^{0}$. This immediately gives vanishing of (B5) for $\xi=0$. Other components of (B5) vanish due to (31) and (B3).

For $\alpha=0$ we have:

$$
\begin{aligned}
& c^{0}(x) D_{00^{\prime} 0^{\prime \prime}}^{(1) 0} c^{0}\left(x^{\prime}\right) c^{0}\left(x^{\prime \prime}\right)=-\frac{1}{2} c^{0}(x) C_{00^{\prime}}^{i} C_{i 0^{\prime \prime}}^{0} c^{0}\left(x^{\prime}\right) c^{0}\left(x^{\prime \prime}\right)=8 c^{0} \partial_{i} c^{0} \partial_{j} c^{0} K^{i j} \delta\left(x, x^{\prime}\right) \delta\left(x^{\prime}, x^{\prime \prime}\right)=0 \\
& c^{0}(x) D_{00^{\prime} 0^{\prime \prime}}^{(1) i} c^{0}\left(x^{\prime}\right) c^{0}\left(x^{\prime \prime}\right)=\frac{1}{2} c^{0}(x)\left\{\Phi_{0}, C_{00}^{i}\right\} c^{0}\left(x^{\prime}\right) c^{0}\left(x^{\prime \prime}\right)=0 \\
& c^{0}(x) D_{00^{\prime} 0^{\prime \prime}}^{(1) p} c^{0}\left(x^{\prime}\right) c^{0}\left(x^{\prime \prime}\right)=-2\left\{c^{0}(x) \Phi^{H}(x), K^{j}\left[c^{0}\left(x^{\prime}\right), c^{0}\left(x^{\prime \prime}\right)\right] A_{0}^{p}\right\}
\end{aligned}
$$

where the first line is zero due to contraction of a symmetric tensor with an antisymmetric one. In the second line we used second of the equations (B3).

To calculate the remaining components of $D^{(1)}$ the following brackets are needed:

$$
\begin{aligned}
\left\{c^{0} \Phi_{0}, K^{j}\left[c^{0}, c^{0}\right] \xi_{j}^{a}\right\}= & 2 c^{0} \partial_{i} c^{0} \partial_{k} c^{0}\left(\left(\widetilde{E}_{a}^{i} \widetilde{E}_{b}^{k}-\widetilde{E}_{a}^{k} \widetilde{E}_{b}^{i}\right) \Phi_{b}^{G}\right. \\
& \left.+\left(\widetilde{E}_{a}^{i} \widetilde{E}_{g}^{k}-\widetilde{E}_{a}^{k} \widetilde{E}_{g}^{i}\right) \varepsilon^{g f b} \chi_{f} \Phi_{b}^{L}\right) \\
\left\{c^{0} \Phi_{0}, K^{j}\left[c^{0}, c^{0}\right]\left(\eta_{j}^{a}+\varepsilon^{a b c} \xi_{j}^{b} \chi_{c}\right)\right\}= & 2 c^{0} \partial_{i} c^{0} \partial_{k} c^{0}\left(-\varepsilon^{a d e} \chi_{d}\left(\widetilde{E}_{e}^{i} \widetilde{E}_{b}^{k}-\widetilde{E}_{e}^{k} \widetilde{E}_{b}^{i}\right) \Phi_{b}^{G}\right. \\
& \left.-\varepsilon^{a d e} \chi_{d}\left(\widetilde{E}_{e}^{i} \widetilde{E}_{g}^{k}-\widetilde{E}_{e}^{k} \widetilde{E}_{g}^{i}\right) \varepsilon^{g f b} \chi_{f} \Phi_{b}^{L}\right)
\end{aligned}
$$

By introducing a $3 \times 6$ matrix field $\widetilde{E}_{p}^{i}=\left(\widetilde{E}_{a}^{i}, \varepsilon_{a b c} \widetilde{E}_{b}^{i} \chi_{c}\right), p=1, \ldots, 6$, one can represent the non-vanishing second order structure functions in an elegant form:

$$
c^{0}(x) U_{00^{\prime} 0^{\prime \prime}}^{(2) p q} c^{0}\left(x^{\prime}\right) c^{0}\left(x^{\prime \prime}\right)=-8 c^{0} \partial_{i} c^{0} \partial_{k} c^{0} \widetilde{E}_{p}^{i} \widetilde{E}_{q}^{k} \delta\left(x, x^{\prime}\right) \delta\left(x^{\prime}, x^{\prime \prime}\right)
$$

Third order structure functions are defined as:

$$
3 U_{\alpha \beta \gamma \delta}^{(3) \xi \eta \lambda} \Phi_{\lambda}=\left(-\left\{U_{\alpha \beta \gamma}^{(2) \xi \eta}, \Phi_{\delta}\right\}-\frac{1}{8}\left\{C_{\alpha \beta}^{\xi}, C_{\gamma \delta}^{\eta}\right\}+\frac{3}{2} C_{\alpha \beta}^{\lambda} U_{\gamma \delta \lambda}^{(2) \xi \eta}+2 U_{\alpha \beta \gamma}^{(2) \xi \lambda} C_{\delta \lambda}^{\eta}\right)_{[\alpha \beta \gamma \delta]}^{[\xi \eta]}
$$

As before, only the functions with $\alpha=\beta=\gamma=0$ could be non-zero. By straightforward calculations one can demonstrate that they vanish as well. There are no non-zero third or higher order structure functions in the HilbertPalatini gravity.

\section{APPENDIX C:}

In this Appendix we prove that imaginary part of the kinetic term of the Ashtekar action (7) vanishes for the fields (42) provided the real canonical variables satisfy the second class constraints (27) and the Gauss and Lorentz constraints.

Consider the kinetic term:

$$
\begin{aligned}
\operatorname{Im} A_{i}^{a} \partial_{t} P_{a}^{i}= & \left(\left(\delta_{a b}\left(1-\chi^{2}\right)+\chi_{a} \chi_{b}\right) \xi_{i}^{b}-\varepsilon^{a b c} \chi_{b} \eta_{i}^{c}\right) \partial_{t} \widetilde{E}_{a}^{i} \\
& -\left(\varepsilon^{a b c} \eta_{i}^{b} \widetilde{E}_{c}^{i}+\chi_{a} \xi_{i}^{b} \widetilde{E}_{b}^{i}-\xi_{i}^{a} \widetilde{E}_{b}^{i} \chi_{b}\right) \partial_{t} \chi_{a}
\end{aligned}
$$

where the expressions (42) were substituted. By making use of the constraints one can rewrite (C1) in the following form.

$$
\begin{aligned}
& \operatorname{Im} A_{i}^{a} \partial_{t} P_{a}^{i}=-\frac{1}{2} \partial_{t} \widetilde{E}_{a}^{i}\left[\varepsilon^{a b c} \widetilde{E}_{b}^{k} \widetilde{E}_{c}^{j}\left(\delta_{g f}-\chi_{g} \chi_{f}\right) \underset{\sim}{E_{i}^{g}} \partial_{k} \underset{\sim j}{E_{j}^{f}}\right.
\end{aligned}
$$

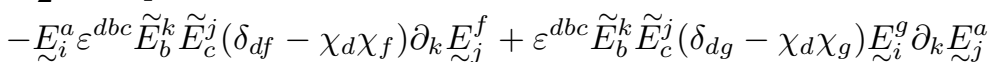

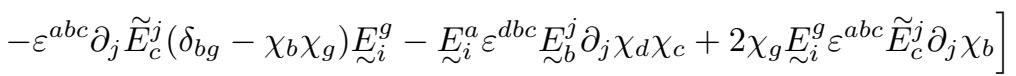

$$
\begin{aligned}
& -\frac{1}{2} \partial_{t} \chi_{a}\left[\varepsilon^{a b c} \widetilde{E}_{b}^{k} \widetilde{E}_{c}^{j} \chi_{g} \partial_{k} \underset{\sim j}{E_{j}^{g}}+\varepsilon^{d b c} \widetilde{E}_{b}^{k} \widetilde{E}_{c}^{j} \chi_{d} \partial_{k} \underset{\sim}{E_{j}^{a}}+\varepsilon^{a b c} \partial_{j} \underset{\sim b}{E_{b}^{j}} \chi_{c}+2 \varepsilon^{a b c} \widetilde{E}_{b}^{j} \partial_{j} \chi_{c}\right] \\
& =\frac{1}{2} \partial_{t}\left(\varepsilon^{d b c} \widetilde{E}_{b}^{k} \widetilde{E}_{c}^{j}\left(\delta_{d g}-\chi_{d} \chi_{g}\right)\right) \partial_{k} E_{\sim}^{g}-\frac{1}{2} \partial_{t} E_{\sim}^{g} \partial_{k}\left(\varepsilon^{d b c} \widetilde{E}_{b}^{k} \widetilde{E}_{c}^{j}\left(\delta_{d g}-\chi_{d} \chi_{g}\right)\right)
\end{aligned}
$$




$$
\begin{aligned}
& +\frac{1}{2} \partial_{t}\left(\widetilde{E}_{a}^{i} \chi_{b}\right) \varepsilon^{a b c} \partial_{i} \chi_{c}-\frac{1}{2} \varepsilon^{a b c} \partial_{t} \chi_{c} \partial_{i}\left(\widetilde{E}_{a}^{i} \chi_{b}\right) \\
= & \frac{1}{2} \partial_{t}\left(\varepsilon^{d b c} \widetilde{E}_{b}^{k} \widetilde{E}_{c}^{j}\left(\delta_{d g}-\chi_{d} \chi_{g}\right) \partial_{k}{\underset{\sim}{E}}_{j}^{g}\right)-\frac{1}{2} \partial_{k}\left(\varepsilon^{d b c} \widetilde{E}_{b}^{k} \widetilde{E}_{c}^{j}\left(\delta_{d g}-\chi_{d} \chi_{g}\right) \partial_{t}{\underset{\sim}{j}}_{j}^{g}\right) \\
& +\frac{1}{2} \partial_{t}\left(\varepsilon^{a b c} \widetilde{E}_{a}^{i} \chi_{b} \partial_{i} \chi_{c}\right)-\frac{1}{2} \partial_{i}\left(\varepsilon^{a b c} \widetilde{E}_{a}^{i} \chi_{b} \partial_{t} \chi_{c}\right)
\end{aligned}
$$

Thus the imaginary part of the kinetic term is a total derivative and can be neglected.

[1] A. Ashtekar, Phys. Rev. Lett. 57, 2244 (1986); Phys. Rev. D 36, 1587 (1987).

[2] S. Melosh, H. Nicolai, Phys. Lett. B416, 91 (1998).

[3] C. Rovelli, Loop Quantum Gravity, electronic journal Living Reviews, gr-qc/9710008.

[4] A. Ashtekar, Lectures on non-perturbative canonical gravity. Notes prepared in collaboration with R. Tate (World Scientific, Singapore, 1991).

[5] P. Peldan, Class. Quantum Grav. 11, 1087 (1994).

[6] A. Ashtekar, P. Mazur and C.G. Torre, Phys. Rev. D36, 2955 (1987).

[7] L.D. Faddeev and V.N. Popov, Phys. Lett. B25, 29 (1967).

[8] M. Henneaux, Phys. Rev. D24, 986 (1983).

[9] J.E. Nelson and C. Teitelboim, Ann. Phys. (NY) 116, 86 (1978).

[10] A. Ashtekar, A.P. Balachandran and S.G. Jo, Int. J. Mod. Phys. A4, 1493 (1989).

[11] J. Samuel, Pramana-J. Phys. 28, L429 (1987);

T. Jacobson and L. Smolin, Class. Quantum Grav. 5, 583 (1988).

[12] I. Bengtsson, Int. J. Mod. Phys. A4, 5527 (1989).

[13] R.P. Wallner, Phys. Rev. D46, 4263 (1992).

[14] A. Ashtekar, J.D. Romano and R.S. Tate, Phys. Rev. D40, 2572 (1989).

[15] G. Immirzi, Class. Quantum Grav. 10, 2347 (1993).

[16] G. Yoneda and H. Shinkai, Class. Quantum Grav. 13, 783 (1996).

[17] P.A.M. Dirac, Lectures on quantum mechanics (Yeshiva University, NY, 1964).

[18] V. Khatsymovsky, Phys. Lett. B 394, 57 (1997).

[19] E.S. Fradkin and G.A. Vilkovisky, Phys. Lett. B55, 244 (1975);

I.A. Batalin and G.A. Vilkovisky, Phys. Lett. B69, 309 (1977);

E.S. Fradkin and T.E. Fradkina, Phys. Lett. B72, 343 (1978).

[20] M. Henneaux, Phys. Rep. 126, 1 (1985).

[21] I. Grigentch and D. Vassilevich, Int. J. Mod. Phys. D4, 581 (1995).

[22] S. Alexandrov, I. Grigentch and D. Vassilevich, Class. Quantum Grav. 15, 573 (1998).

[23] L.D. Faddeev, Teor. Mat. Fiz. 1, 3 (1969).

[24] T. Thiemann, Class. Quantum Grav. 13, 1383 (1996), A. Ashtekar, Phys. Rev. D53, 2865 (1996). 